\title{
Rapid purification of sub-micrometer particles for enhanced drug release and microvesicles isolation
}

\author{
Hui Min Tay ${ }^{1}$, Sharad Kharel², Rinkoo Dalan ${ }^{1,3}$, Zhijie Joshua Chen ${ }^{2}$, Kah Kee Tan², Bernhard O Boehm ${ }^{1,3,4}$, \\ Say Chye Joachim Loo $^{2,5}$ and Han Wei Hou ${ }^{1}$
}

\begin{abstract}
Efficient separation of sub-micrometer synthetic or biological components is imperative in particle-based drug delivery systems and purification of extracellular vesicles for point-of-care diagnostics. Herein, we report a novel phenomenon in spiral inertial microfluidics, in which the particle transient innermost distance $\left(D_{\text {inner }}\right)$ varies with size during Dean vortices-induced migration and can be utilized for small microparticle (MP) separation; aptly termed as high-resolution Dean flow fractionation (HiDFF). The developed technology was optimized using binary bead mixtures $(1-3 \mu \mathrm{m})$ to achieve $\sim 100$ - to 1000 -fold enrichment of smaller particles. We demonstrated tunable size fractionation of polydispersed drug-loaded poly(lactic-co-glycolic acid) particles for enhanced drug release and anti-tumor effects. As a proof-of-concept for microvesicles studies, circulating extracellular vesicles/ MPs were isolated directly from whole blood using HiDFF. Purified MPs exhibited well-preserved surface morphology with efficient isolation within minutes as compared with multi-step centrifugation. In a cohort of type 2 diabetes mellitus subjects, we observed strong associations of immune cell-derived MPs with cardiovascular risk factors including body mass index, carotid intima-media thickness and triglyceride levels $(\boldsymbol{P}<0.05)$. Overall, HiDFF represents a key technological progress toward highthroughput, single-step purification of engineered or cell-derived MPs with the potential for quantitative MP-based health profiling.
\end{abstract}

NPG Asia Materials (2017) 9, e434; doi:10.1038/am.2017.175; published online 29 September 2017

\section{INTRODUCTION}

Enabling technologies for continuous, size-based separation of submicrometer engineered or biological components are highly desirable in clinical applications, such as particle-based drug delivery systems ${ }^{1}$ and the purification of extracellular vesicles in clinical diagnostics. ${ }^{2}$ In microparticle fabrication, conventional 'bottom-up' self-assembly emulsification techniques yield a broad particle size distribution, which can affect the drug release kinetics and biotransport in blood., ${ }^{3,4}$ Although well-controlled and monodisperse particles can be produced by 'top-down' approaches using specific lithographic techniques ${ }^{5,6}$ and microfluidics, ${ }^{7,8}$ microfabricated particles are prone to damage during mechanical harvesting, a problem further aggravated at the smaller/nanoscale level. Similarly, microfluidic synthesis of drug-loaded polymeric particles requires compatible drug/surfactant chemistry with additional steps to remove solvent prior use. Developing novel tools to achieve tunable size fractionation of polydispersed synthetic particles would enable optimal biodistribution and controlled drug release. Such technologies also facilitate physical isolation of smaller biological targets $(<2 \mu \mathrm{m})$ including platelets, microbes and extracellular vesicles in a label-free manner for unbiased downstream analysis.
In recent years, extracellular vesicles have emerged as key mediators for intercellular communication and pathophysiological responses, which can be used for clinical diagnostics and therapeutics. ${ }^{9,10}$ Depending on the biogenesis, they are termed as exosomes or microvesicles based on their endosomal or plasma membrane origin, respectively. Microvesicles or microparticles (MPs) are $\sim 0.1-1 \mu \mathrm{m}$ in size and carry a multitude of proteins, mRNA and microRNA cargo. Traditional exosome/MPs isolation using high speed- or ultracentrifugation is time-consuming $(\sim 2-5 \mathrm{~h})$ and subjected to significant variability. ${ }^{11}$ Immunoaffinity capture is more effective, ${ }^{12}$ but this process carries the risk of losing MPs functionalities after elution, which advocates the need for label-free approaches. Notably, isolation of blood-borne MPs remains a significant technical challenge due to the high cellular components in blood $(\sim 50 \% \mathrm{v} / \mathrm{v})$ and the similar sizes of MPs and platelets $(\sim 2-3 \mu \mathrm{m}) .{ }^{13}$ Microfluidic MPs isolation from blood have been demonstrated ${ }^{14-16}$ but are limited by low throughput $\left(\sim 0.1-2 \mu \mathrm{min}^{-1}\right)$ and on-chip molecular analysis, as purified MPs remain captured inside these devices. Complicated protein patterning or multiplexed binding methods are often employed in clinical testing to isolate MPs with large surface heterogeneity or a lack of well-defined surface markers. ${ }^{16}$ In this

\footnotetext{
${ }^{1}$ Lee Kong Chian School of Medicine, Nanyang Technological University, Singapore, Singapore; ${ }^{2}$ School of Materials Science and Engineering, Nanyang Technological University, Singapore, Singapore; ${ }^{3}$ Department of Endocrine and Diabetes, Tan Tock Seng Hospital, Singapore, Singapore; ${ }^{4}$ Imperial College London, London, UK and ${ }^{5}$ Singapore Centre for Environmental Life Sciences Engineering, Singapore, Singapore

Correspondence: Dr HW Hou, Lee Kong Chian School of Medicine, Nanyang Technological University, Clinical Sciences Building, Level 11, 11 Mandalay Road, Singapore 308232, Singapore.

E-mail: hwhou@ntu.edu.sg

Received 28 March 2017; revised 11 July 2017; accepted 13 July 2017
} 
regard, it is crucial to develop new strategies for continuous, label-free MPs purification from whole blood to facilitate off-chip downstream analysis, as well as integration into point-of-care testing platforms.

Microfluidic technologies have been widely used for large $(>10 \mu \mathrm{m})$ particle and cell separation ${ }^{17}$ but are explored relatively less in the smaller particle size region $(\sim 1-2 \mu \mathrm{m}) .^{18-20}$ Inertial microfluidics is a promising separation technique that involves the lateral migration of particles or cells across streamlines to focus at distinct positions due to dominant inertial forces $\left(\mathrm{F}_{\mathrm{L}}\right)$ at high flow rates (Reynolds number, Re $\sim 50-100) .{ }^{21}$ Our group has previously developed an inertial microfluidics spiral sorter (Dean flow fractionation, DFF $)^{22}$ that takes advantage of additional secondary Dean vortices for well-controlled, Dean-induced migration of small particles including bacteria, ${ }^{23}$ nanoparticles ${ }^{24}$ and biomolecules ${ }^{25}$ to channel outer wall, whereas target cells $(>10 \mu \mathrm{m})$ are focused inertially near the inner wall for separation. However, a major drawback of this method is its inability to further size-fractionate smaller MPs (for example, $1 \mu \mathrm{m}$ vs $2 \mu \mathrm{m}$ ), as they recirculate continuously due to the Dean vortices. Herein, we report a novel Dean migration phenomenon in spiral microchannels for small MP separation with superior resolution, aptly termed as high-resolution DFF (HiDFF). Contrary to current inertial microfluidics-based devices, the developed technology achieves sub-micrometer particle separation based on the differential particle innermost transient position $\left(D_{\text {inner }}\right)$ at the channel inner wall during lateral migration (Figures $1 \mathrm{a}$ and $\mathrm{b}$ ). As particle inertial focusing is not necessary, channel dimensions are increased to minimize clogging while significantly improving the throughput $\left(\sim 100 \mu \mathrm{min}^{-1}\right)$. We first applied the HiDFF technology in particlebased drug delivery system to achieve tunable size fractionation of drug-loaded particles (Figure 1c). Engineered sub-micrometer drugloaded poly(lactic-co-glycolic acid) (PLGA) particles $(<1 \mu \mathrm{m})$ were purified efficiently from a polydisperse particle mixture $(0.1-10 \mu \mathrm{m})$, which exhibited enhanced drug release and inhibited breast cancer cell (MCF-7) proliferation in vitro. To validate our method for microvesicles applications, we combined HiDFF and flow cytometry to isolate and phenotype circulating MPs directly from whole blood (Figure 1d). The HiDFF-purified MPs were continuously eluted offchip in a single step and their surface morphology was well preserved with fewer 'breakages' compared with high-speed centrifugation. Similar quantities of endothelial-derived and immune cell-derived MPs were isolated using HiDFF in a significantly simpler and faster ( $\sim$ minutes) operation, as compared with centrifugation ( $\sim$ hours). Finally, we clinically validated the developed technology in a cohort of patients with type 2 diabetes mellitus (T2DM) and observed that immune cell-derived MPs were strongly correlated with established cardiovascular risk factors, including body mass index, carotid intimamedia thickness and triglyceride levels $(P<0.05)$. Taken together, these results clearly demonstrate the capabilities of HiDFF for highthroughput separation of small engineered or biological targets and can be further developed into a clinical tool for rapid quantitative assessment of immune and vascular health using MPs.

\section{MATERIALS AND METHODS}

\section{Microdevice fabrication}

The HiDFF device was fabricated in polydimethylsiloxane (Dow Corning, Midland, MI, USA) using standard soft lithography methods. Polydimethylsiloxane prepolymer was mixed in a 10:1 ratio (w/w) with curing agent and poured over a patterned silicon wafer. The polydimethylsiloxane mixture was then cured at $80^{\circ} \mathrm{C}$ for $2 \mathrm{~h}$ and peeled from the wafer. Inlet and outlet holes $(1.5 \mathrm{~mm})$ were punched using a biopsy puncher and the devices were cleaned thoroughly with isopropanol before bonding to $1 \mathrm{~mm}$-thick glass slides using an air plasma machine (Harrick Plasma, Inc., Ithaca, NY, USA).

\section{Device characterization}

Fluorescent polystyrene beads (Bangs Laboratories, Fishers, IN, USA) of defined sizes $(50 \mathrm{~nm}, 1 \mu \mathrm{m}, 2 \mu \mathrm{m}$ and $3 \mu \mathrm{m})$ were pumped into the sample inlet at different flow rates $\left(70-100 \mu \mathrm{lmin}^{-1}\right)$ using a syringe pump (Chemyx Inc., Stafford, TX, USA), whereas sheath buffer (phosphate-buffered saline (Lonza, Basel, Switzerland) supplemented with $0.1 \%$ bovine serum albumin (Biowest, Nuaillé, France)) was injected into the sheath inlet via a separate syringe pump. The ratio of sample to sheath flow rate was fixed at 1:5 to confine the sample stream at the outer wall. The device was mounted on a Nikon Eclipse Ti inverted phase-contrast microscope (Nikon Instruments Inc., Melville, NY, USA) equipped with Metamorph software (Molecular Devices, Sunnyvale, CA, USA) and was used to visualize the equilibrium positions of different bead sizes based on fluorescence imaging. Phase-contrast high-speed videos (10 $000 \mathrm{fps})$ were captured using a Phantom V9.1 high-speed camera (Vision Research, Wayne, NJ, USA).

\section{Cell culture}

MCF-7, a human breast cancer cell line, was cultured in Dulbecco's modified Eagle's medium (HyClone, Logan, UT, USA) supplemented with $10 \%$ fetal bovine serum (Gibco, Grand Island, NY, USA) and 1\% penicillin/streptomycin (Gibco). Human umbilical vascular endothelial cells were cultured in endothelial cell growth media (EGM-2; Lonza) supplemented by $10 \%$ fetal bovine serum and $1 \%$ penicillin/streptomycin. The cells were maintained at $37^{\circ} \mathrm{C}$ in a humidified $5 \% \mathrm{CO}_{2}$ incubator and passaged every 4 days. Confluent cell layers were dissociated using $0.25 \%$ trypsin with $1 \mathrm{~mm}$ EDTA (Gibco).

\section{Fabrication of PTX-loaded PLGA MPs}

Paclitaxel (PTX)-loaded PLGA MPs were prepared using an (oil/water) emulsion solvent evaporation technique. Briefly, $1 \mathrm{~g}$ of PLGA (intrinsic viscosity: 1.18, Purac, Corbion, Amsterdam, Netherlands) and $50 \mathrm{mg}$ of PTX (5\% w/w theoretical drug loading) were added in $20 \mathrm{ml}$ of dichloromethane. The mixture was put under magnetic stirring for $2 \mathrm{~h}$ to completely dissolve the PLGA and PTX in the solvent. Next, the drug-polymer solution (oil phase) was emulsified with $75 \mathrm{ml}$ of polyvinyl alcohol aqueous solution $(3 \% \mathrm{w} / \mathrm{v})$ (water phase) using a T-18 Ultra Turrax digital homogenizer (IKA, Wilmington, NC, USA) set at 7000 r.p.m. for $30 \mathrm{~min}$. The resulting (oil/water) emulsion was then added to $100 \mathrm{ml}$ of polyvinyl alcohol solution $(3 \% \mathrm{w} / \mathrm{v})$ and kept under magnetic stirring overnight to allow for the residual dichloromethane to evaporate. Finally, the particles were washed three times with deionized water, pelleted by centrifugation at 9000 r.p.m. for $10 \mathrm{~min}$ and freeze-dried overnight. See Supplementary Information for more details on MPs scanning electron microscopy (SEM) imaging, drug encapsulation and drug-release characterization.

\section{Size fractionation of PTX-loaded PLGA MPs}

The fabricated PTX-loaded PLGA MPs were size fractionated into three different size groups (large, medium and small) using a two-step HiDFF separation approach. Deionized water was used as sheath buffer to facilitate SEM imaging. At the first stage, large particles $(>5 \mu \mathrm{m})$ were sorted from the polydisperse mixture into outlet 1 at a sample flow rate of $\sim 160 \mu \mathrm{l} \mathrm{min}{ }^{-1}$. The eluent collected from outlet 2 was subjected to another HiDFF separation (second stage) at a sample flow rate of $80 \mu \mathrm{min}^{-1}$ to fractionate the medium and small particles into outlet 2 and outlet 1, respectively. See Supplementary Information on cell proliferation assay.

\section{Isolation of MPs from whole blood}

Whole-blood samples obtained from healthy donors and T2DM patients were diluted $(1: 5 \mathrm{v} / \mathrm{v})$ with a buffer (phosphate-buffered saline with $0.1 \%$ bovine serum albumin) and fractionated using the HiDFF device at a sample flow rate of $80 \mu \mathrm{min}^{-1}$. To compare the MPs recovery, conventional multi-step centrifugation was used to process the same blood volume as HiDFF. Briefly, whole blood was spun down at $2000 \mathrm{~g}$ for $20 \mathrm{~min}$ to remove cell components and the supernatant was centrifuged again at $20000 \mathrm{~g}$ for $45 \mathrm{~min}$ to pellet the MPs. 
a

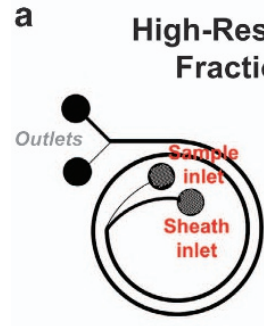
ractionation (HiDFF)

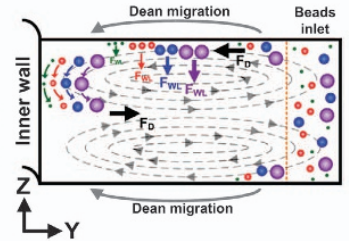

C
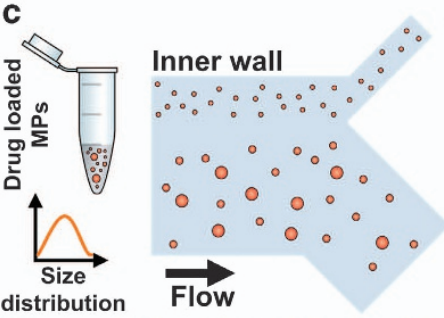

Small MPs

$(<1 \mu \mathrm{m})$

Large MPs

$(\sim 1-10 \mu \mathrm{m})$

b

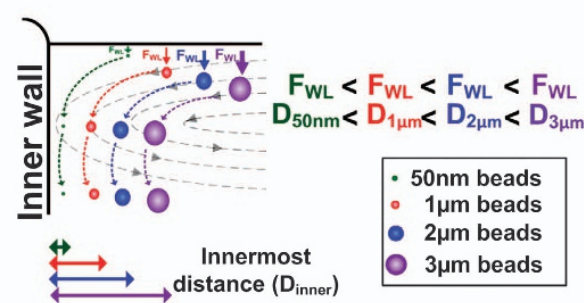

d

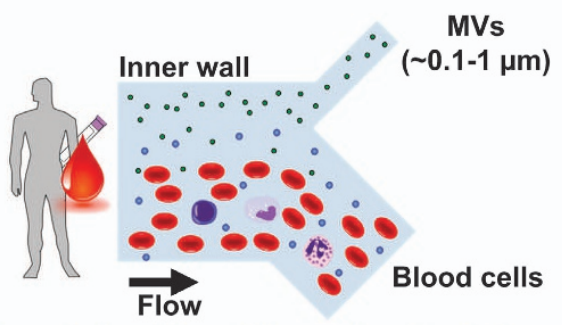

Vascular health profiling using circulating microvesicles (MVs)

f

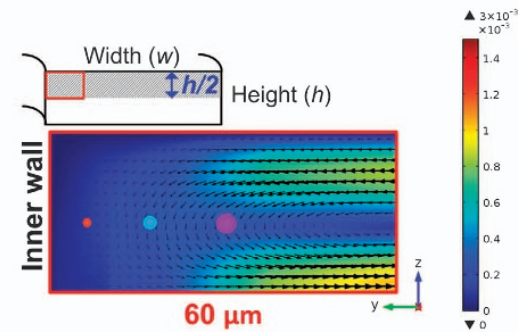

Figure 1 Particle separation using HiDFF. (a) Schematic illustration of the HiDFF separation principle in a two-inlet, two-outlet spiral microchannel. Particles introduced at the outer wall migrate laterally towards the inner wall under the influence of Dean vortices and the Dean drag forces $\left(F_{\mathrm{D}}\right)$. As the particles migrate along the channel top and bottom, they experience size-dependent, wall-induced lift forces $\left(F_{\mathrm{WL}}\right)$ that push larger particles further away from the surfaces. (b) Subtle differences in the particle $z$-position (along the height) lead to a size-based transient innermost distance $\left(D_{\text {inner }}\right)$ at the inner wall, which can be exploited for small particle separation with superior resolution. (c) Size fractionation of polydispersed drug-loaded MPs using HiDFF to purify submicrometer particles for enhanced drug delivery. (d) Single-step isolation of circulating extracellular microvesicles/MPs in blood for rapid vascular health profiling. (e) Composite high speed images at the channel bottom ( $\sim 5 \mu \mathrm{m}$ height) indicate increasing axial flow speed with particle size. (f) Fluid simulation (COMSOL) of the transverse Dean flow patterns of the channel top half inner region. Color bar represents the Dean velocity magnitude (in $\mathrm{m} \mathrm{s}^{-1}$ ). Empirically determined $D_{\text {inner }}$ positions of 1,2 and $3 \mu \mathrm{m}$ beads are indicated (drawn to scale).

\section{Characterization of MPs}

Dynamic light scattering measurements of the particle sizes were performed in triplicates using the Zetasizer Nano ZS (Malvern Instrument, Malvern, UK) with MP samples adequately diluted in filtered distilled water. See Supplementary Information for more details on field-emission SEM imaging and nanoparticle tracking analysis.

\section{Flow cytometry}

All antibodies were purchased from eBioscience (San Diego, CA, USA), and flow cytometry analysis was performed using a BD LSR Fortessa flow cytometer (BD Biosciences, San Jose, CA, USA). For HiDFF and centrifugation comparative studies, $1 \mathrm{ml}$ of whole blood was processed individually and the purified MPs were resuspended to an equal volume of $250 \mu$. The sorted MPs from whole blood were stained for $30 \mathrm{~min}$ at $4{ }^{\circ} \mathrm{C}$ (1:20 dilution) with allophycocyanin (APC)-labeled anti-human vascular endothelial (VE)-cadherin and fluorescein isothiocyanate-labeled antihuman CD41a to identify endothelial-derived MPs (EMPs), fluorescein isothiocyanate-labeled antihuman $\mathrm{CD} 3$ and allophycocyanin-labeled anti-human CD19 to identify T- and B-lymphocyte-derived MPs, fluorescein isothiocyanate-labeled antihuman CD45 to identify leukocyte-derived MPs (LMPs), and allophycocyaninlabeled anti-human CD66b to identify neutrophil-derived MPs (NMPs). $3 \mu \mathrm{m}$ beads were added to the purified MPs samples prior data acquisition and the MPs count was evaluated based on the detection gate of 15000 beads.

\section{Study approval}

For all subjects, written informed consent was obtained during recruitment. All protocols were approved by the institutional review boards of Nanyang Technological University (IRB-2014-04-27) and Tan Tock Seng Hospital (2014100416), where the studies were conducted. A total of nine subjects with Chinese or Indian ethnicity were recruited. For fingerprick blood sampling, blood was obtained from healthy donors using a disposable lancet (Roche Diagnostics Corp., Basel, Switzerland) and collected in EDTA tubes (BD Microtainer). For blood sampling by venipuncture, $3 \mathrm{ml}$ of blood was collected into a EDTA vacutainer (BD Biosciences) and shipped to Nanyang Technological University on the same day for microfluidics experiments and flow cytometry analysis.

\section{Statistical analysis}

All numerical data were expressed as the mean \pm s.d., unless otherwise specified. We assessed the statistical significance of the difference between two sets of data using the Mann-Whitney $U$-test (unless otherwise specified) with $P<0.05$ considered to be of significant difference. All analysis was performed using GraphPad Prism V5.0 (GraphPad Software, La Jolla, CA, USA).

\section{RESULTS}

\section{MPs separation using HiDFF}

The developed HiDFF microdevices were fabricated in polydimethylsiloxane using standard soft lithography. The design consists of a two- 

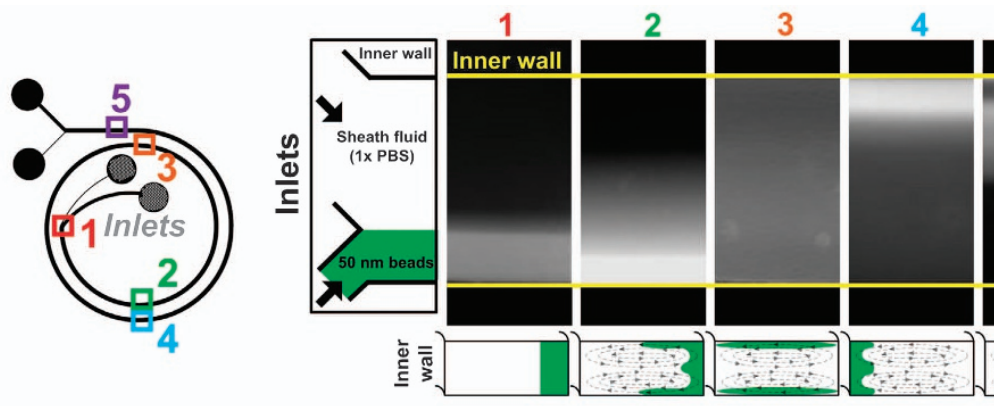

5

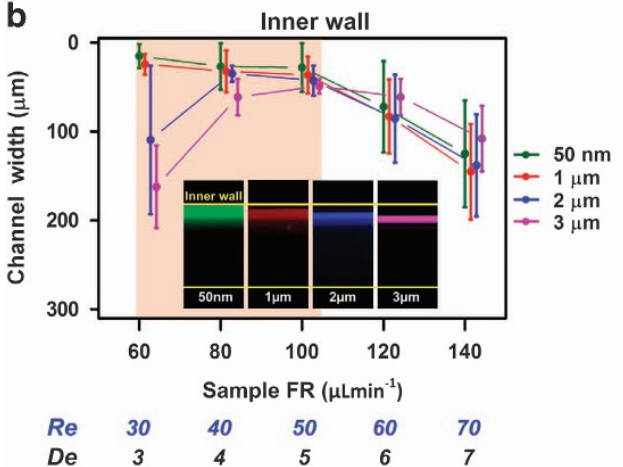

C

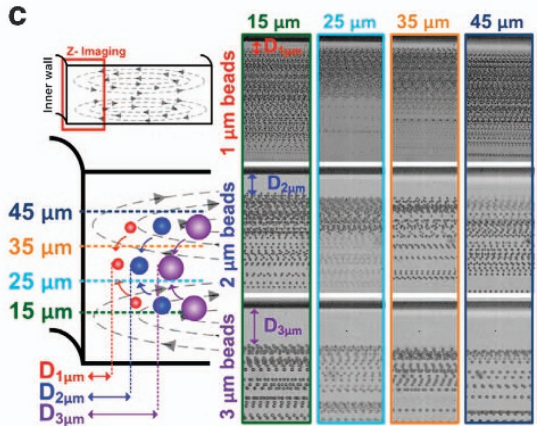

d

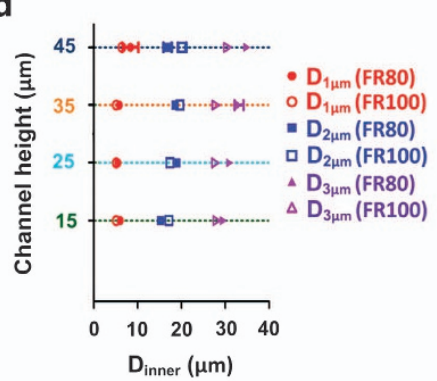

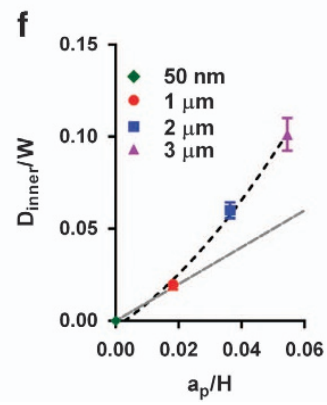

Figure 2 Superior separation resolution of HiDFF. (a) Average fluorescent composite images and a cross-sectional channel schematic illustrating the migration trajectory of $50 \mathrm{~nm}$ particles along the microchannel. (b) Characterization of the Dean migration profile for different particle sizes. The inset fluorescent composite images illustrate the distinct innermost distance $\left(D_{\text {inner }}\right)$ for different sized particles. The highlighted region represents the working flow conditions for the HiDFF. High speed Z-imaging ( $\times 40$ magnification) of the inner wall region at different planes indicate similar $D_{\text {inner }}$ along (c) the channel height and (d) at different flow conditions. (e) The effect of particle size on the Dean migration velocity at varying sample flow rates. (f) The relationship between the $D_{\text {inner }}$ and particle size. The data are fitted with non-linear regression (black dashed line, $R^{2}: 0.9879$ ) and the gray line represents unity.

inlet, two-outlet spiral microchannel $(300 \mu \mathrm{m}(\mathrm{w}) \times 55 \mu \mathrm{m}(\mathrm{h}))$ with a radius of $0.5-0.6 \mathrm{~cm}$ (Figure 1a). The magnitude of the Dean vortices is determined by the Dean number $\left(\mathrm{De}=\operatorname{Re}\left(D_{\mathrm{h}} / 2 R\right)^{0.5}\right)$, where $D_{\mathrm{h}}$ is the microchannel hydraulic diameter (m) defined as $2 w \times h /(w+h), R$ is the radius of curvature $(\mathrm{m})$ and $\mathrm{Re}$ is Reynolds number that compares the inertial forces to viscous forces. Based on the Dean velocity equation $\left(U_{\mathrm{De}}=1.84 \times 10^{-4} \mathrm{De}^{1.63}\left(\mathrm{~ms}^{-1}\right)\right)$ from Ookawara et al. ${ }^{26}$ a minimum channel length of $\sim 5.9 \mathrm{~cm}$ is required for Dean migration distance of $300 \mu \mathrm{m}$ (outer to inner wall is approximately the channel width) at Re 50. Hence, the total channel length was fixed at $6.5 \mathrm{~cm}$ in our study and the sample $(50 \mu \mathrm{m}$ wide $)$ and sheath $(250 \mu \mathrm{m}$ wide) inlets were positioned at the outer and inner wall of the channel, respectively. Small MPs (ratio of particle size $\left(a_{\mathrm{p}}\right)$ to $D_{\mathrm{h}}, a_{\mathrm{p}} / D_{\mathrm{h}}<0.07$ ) introduced at the channel outer wall experienced Dean drag forces $\left(F_{\mathrm{D}}\right)$ due to the Dean vortices and migrated laterally towards the inner wall. As they migrated along the channel top or bottom, particles near the surface experienced size-dependent, wall-induced inertial lift forces $\left(F_{\mathrm{WL}} \alpha a_{\mathrm{p}}{ }^{2}\right)$ due to the asymmetrical wake around them that pushed the particles away from the surface. ${ }^{27}$ Hence, the particles flowed at different fluid streamlines, which led to a differential transient innermost position $\left(D_{\text {inner }}\right)$ at the inner wall before they recirculated back toward the outer wall (Figure 1b). Smaller particles (with smaller $D_{\text {inner }}$ ) were positioned closer to the inner wall and were separated into the inner outlet (outlet 1), whereas larger particles were sorted into the outer outlet (outlet 2). Using high-speed imaging $(\sim 10000 \mathrm{fps})$ near the channel bottom $(\sim 5 \mu \mathrm{m}$ from the bottom surface), we observed that the larger particles were flowing at higher axial speeds due to Poiseulle flow profile, as evidenced from the increased particle spacing in the overlaid images, thus indicating that the particles were further away from channel bottom (Figure 1e). Fluid simulation (COMSOL) further confirmed that the transverse Dean flow trajectories based on the subtle changes in the particle $z$-position (distance from surface) could affect the $D_{\text {inner }}$ during Dean migration (Figure 1f).

To ensure negligible inertial effects, we used $50 \mathrm{~nm}$ fluorescent beads to visualize the Dean migration profile at different positions along the microchannel. We also fabricated and tested another microdevice with the same spiral geometry with the exception that 
the sample inlet was positioned at the inner wall instead. Beads introduced at the outer inlet migrated along the channel top and bottom toward the inner wall and recirculated outwards along the channel midline (Figure 2a). In contrast, beads introduced at the inner inlet migrated along the channel midline tightly as a band toward the outer wall, which was consistent with the lateral flow direction of the secondary Dean vortices (Supplementary Figure S1). These differences in the bead lateral positions were used to calculate the Dean migration velocities of the particles and were in good agreement with the analytical values, based on Ookawara et al. ${ }^{26}$ Dean velocity equation and fluid simulations (Supplementary Figures S1 and S2).

To characterize the dependence of $D_{\text {inner }}$ on the particle size, beads of smaller diameters $(50 \mathrm{~nm}, 1 \mu \mathrm{m}, 2 \mu \mathrm{m}$ and $3 \mu \mathrm{m}$ ) were used so that they would not undergo inertial focusing in the device $\left(a_{\mathrm{p}} / h<0.07\right)$. As shown in Supplementary Figure $2 b$, all particles recirculated towards the inner wall and then back to the outer wall with increasing flow rates $(\operatorname{Re} \sim 30-70)$, indicating dominant Dean migration. At lower flow conditions $(\operatorname{Re} \sim 30-50)$, larger particles migrated towards the inner wall at a slightly slower speed due to size-dependent Stoke's drag $\left(\sim a_{\mathrm{p}}\right)$. In addition, $50 \mathrm{~nm}$ beads migrated completely toward the inner wall, whereas larger beads exhibited increasing $D_{\text {inner }}$. For all for bead sizes, there were negligible differences in their Dean migration toward the outer wall at higher flow conditions ( $\operatorname{Re} \sim 60-70)$. Hence, the optimal working conditions for HiDFF separation were set at the lower sample flow rates of 60-100 $\mu \mathrm{l} \mathrm{min}^{-1}$.

Next, we performed high-speed imaging at the inner wall region to further understand the distinct differences in $D_{\text {inner }}$ by visualizing the particle flow position at different planes along the channel height. Composite bright-field images clearly indicated increasing $D_{\text {inner }}$ with particle size $(\sim 5.2 \mu \mathrm{m}$ for $1 \mu \mathrm{m}$ beads, $17.4 \mu \mathrm{m}$ for $2 \mu \mathrm{m}$ beads and $27.8 \mu \mathrm{m}$ for $3 \mu \mathrm{m}$ beads). This trend was similar for different channel heights (Figure 2c) and at higher flow rates (Figure 2d). Interestingly, although the particle $(50 \mathrm{~nm}$ to $3 \mu \mathrm{m})$ varied by few orders of magnitude in size, their Dean migration velocities were similar, with $\sim 2$-fold differences between the $50 \mathrm{~nm}$ and $3 \mu \mathrm{m}$ particles, and negligible differences between $50 \mathrm{~nm}$ and $1 \mu \mathrm{m}$ particles (Figure 2e). We characterized the $D_{\text {inner }}\left(D_{\text {inner }} / w\right)$ and particle size $\left(a_{\mathrm{p}} / h\right)$ and found that the experimental data were in good agreement with the nonlinear regression $\left(R^{2}: 0.99\right)$ as $F_{\mathrm{WL}} \propto a_{\mathrm{p}}{ }^{2}$ based on Saffman lift equation $^{27}$ (Figure $2 \mathrm{f}$ ).

Size fractionation of drug-loaded MPs for enhanced drug release In particle-based engineering for drug delivery systems, particle size is known to affect the physicochemical characteristics, including the drug release profiles, degradation kinetics and biotransport. ${ }^{28,29}$ However, most bottom-up synthesis approaches yield a wide size range of particles and it is challenging to obtain particles of well-defined sizes without additional sample preparation steps. Using PTX and cancer therapy as a model system to demonstrate enhanced drug release using sub-micrometer drug carriers, we size-fractionated PTX-loaded polydisperse MPs using HiDFF and assessed their efficacy in drug release and cancer treatment.

To determine whether the subtle differences in $D_{\text {inner }}$ during the Dean migration can be exploited for separating particles with closely spaced sizes, binary bead mixtures ( 2 and $3 \mu \mathrm{m}$ beads, and 1 and $2 \mu \mathrm{m}$ beads) were processed by the HiDFF device at different sample to sheath flow ratio. As the sample stream was 'pinched' with increasing sheath flow at the inlet, the fluorescent intensity line scan indicated a decrease in the effective width of the particle flow position while the dominant peak at the inner wall remained unchanged at the outlet region. A comparison of the normalized fluorescence intensity distribution also showed an overall tighter bead distribution at higher flow ratios (Supplementary Figure S3). We hypothesized that this was due to smaller variations in the particle initial $y$-position (along the channel width), which enabled them to migrate laterally as a tight band toward the inner wall. Hence, the separation efficiency a

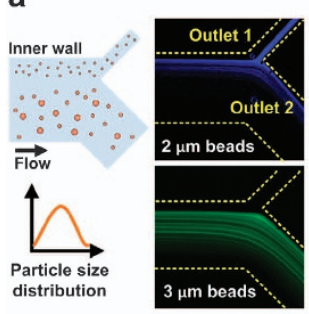

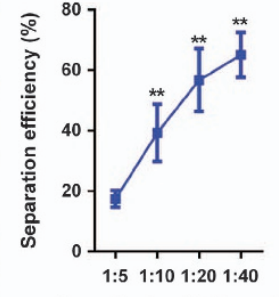

Sample:sheath flow ratio

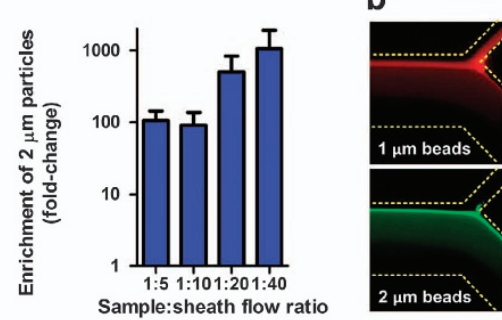

d

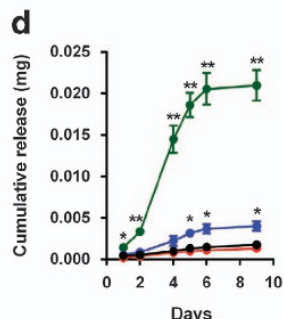

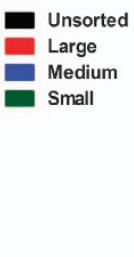
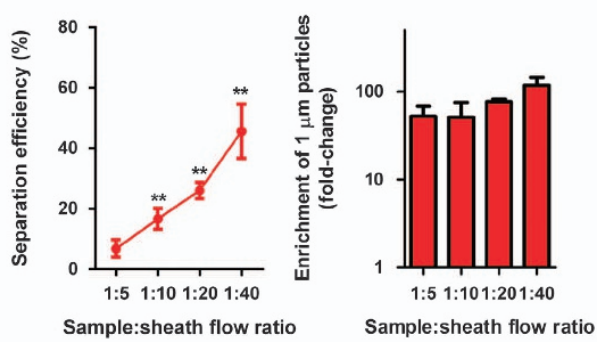

c

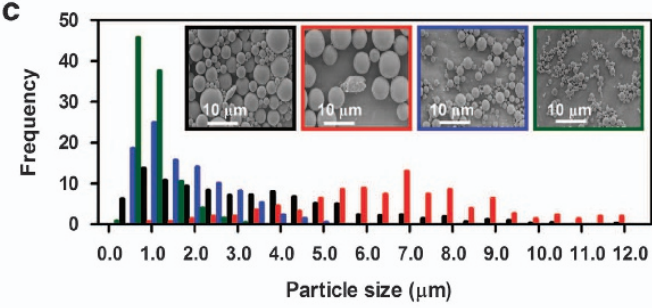

e

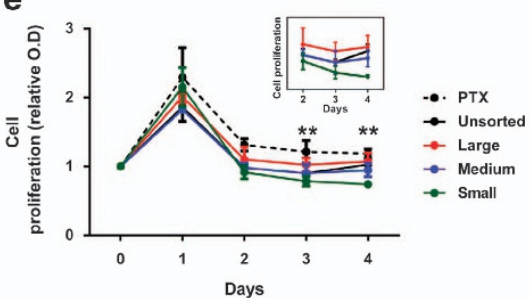

Figure 3 Tunable size fractionation of drug-loaded PLGA MPs using HiDFF. The average fluorescent composite images and separation performance of smaller particles for different sample conditions: sheath flow ratio for (a) $2 \mu \mathrm{m}$ and $3 \mu \mathrm{m}$ binary bead mixtures. (b) $1 \mu \mathrm{m}$ and $2 \mu \mathrm{m}$ binary bead mixtures. Yellow dotted lines indicate the positions of the channel outlet bifurcation. The data are presented as the mean \pm s.d. from $n=4$. ${ }^{* *} P<0.01$ vs control (1:5). (c) Size distribution plot of the PLGA MPs after the two-step HiDFF separation into three sizes (large $(>5 \mu \mathrm{m})$, medium $(2-5 \mu \mathrm{m})$ and small $(<2 \mu \mathrm{m})$ ). Inset SEM images highlight the distinct size differences between samples (inlet) and different size groups. (d) The drug release profile of the fractionated PTXloaded PLGA MPs. (e) The cell proliferation (cell counting kit-8) assay on breast cancer cells (MCF-7) incubated with PTX-loaded PLGA MPs. Inset plot indicates a significant decrease in the relative optical density (OD) for the smallest particle size group. The data are presented as the mean \pm s.d. from $n=3$. ${ }^{*} P<0.05$ and ${ }^{*} P<0.01$ vs unsorted. 
significantly improved from 20 to $60 \%$ for $2 \mu \mathrm{m}$ bead isolation $(2 \mu \mathrm{m}$ and $3 \mu \mathrm{m}$ bead mixture) and from 5 to $40 \%$ for $1 \mu \mathrm{m}$ bead isolation ( 1 and $2 \mu \mathrm{m}$ bead mixture) at a higher sheath flow ( $P<0.01$ vs $1: 5$ ratio), resulting in an enrichment of the smaller particles of $\sim 1000$ - and 100 fold, respectively (Figures 3a and b). Next, we fabricated PLGA MPs containing PTX $(5 \% \mathrm{w} / \mathrm{w})$ using an emulsion solvent evaporation technique ( $58 \%$ encapsulation efficiency, see Methods section for details) for tunable size-based particle fractionation using HiDFF. Using a two-step separation approach, the PTX-loaded PLGA MPs $(0.1-10 \mu \mathrm{m})$ were fractionated into three different size groups (large, medium and small) with different flow rates using the same HiDFF device. In the first step, large particles from the sample mixture were

a
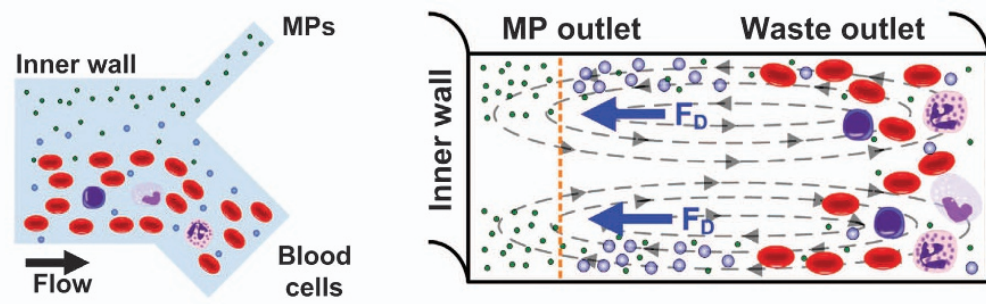
$\because$ Microvesicles/microparticles \& Platelets

\section{Red blood cells (RBCs)} Leukocytes

b
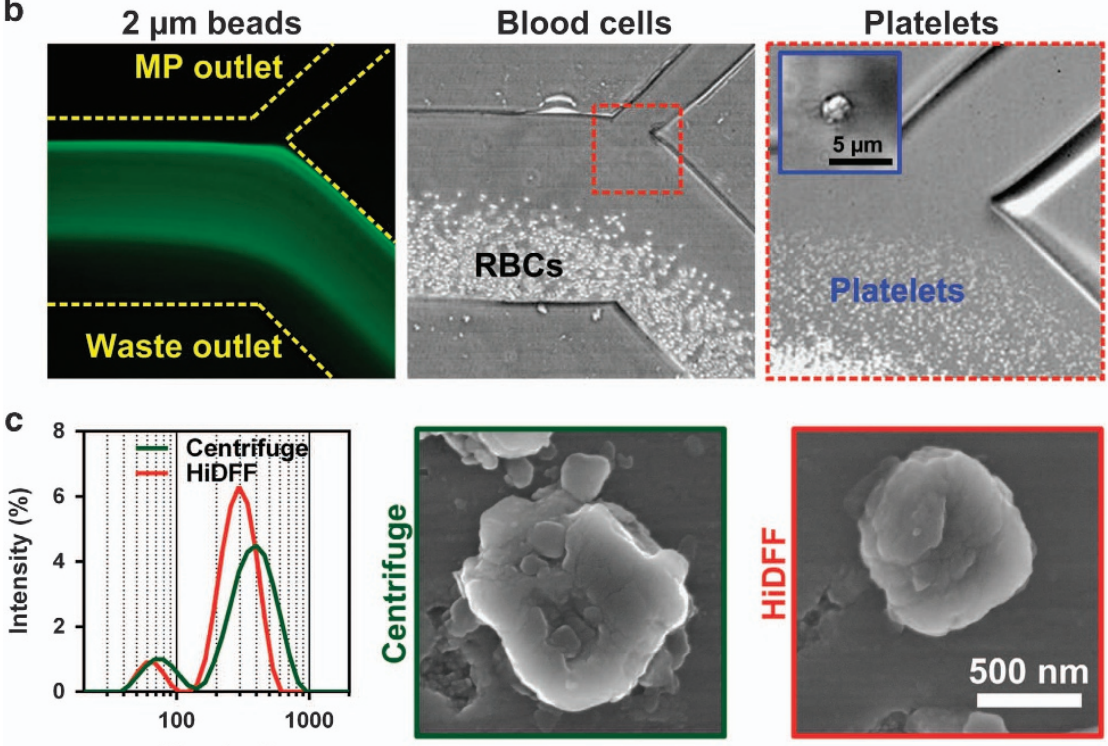

Size $(n m)$
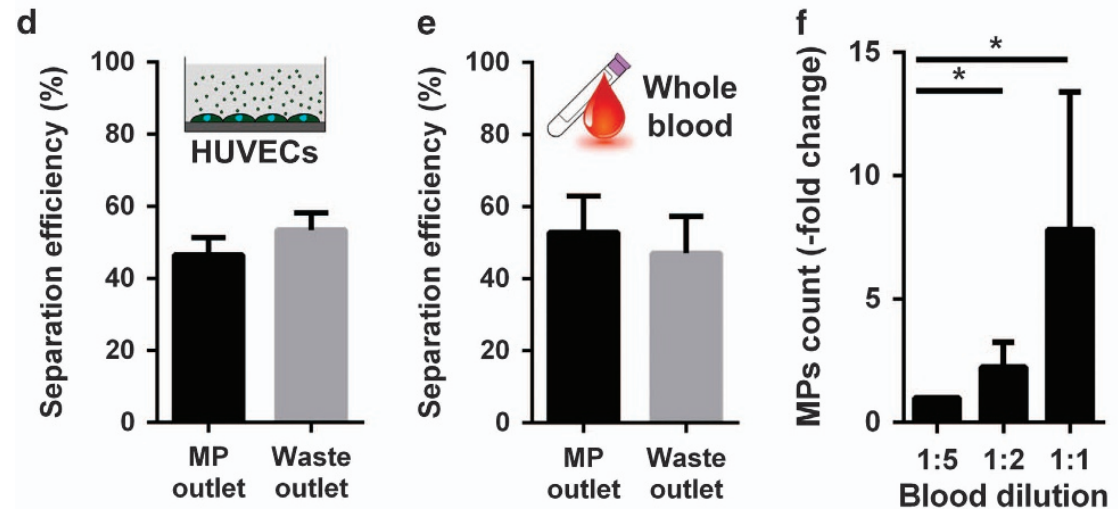

Figure 4 Isolation of circulating MPs from whole blood using HiDFF. (a) Illustration of MPs $(0.1-1 \mu \mathrm{m})$ and platelets $(\sim 2-3 \mu \mathrm{m})$ experiencing the Dean drag forces $\left(F_{\mathrm{D}}\right)$ and migrate towards the inner wall. The smaller MPs recirculate closer to the inner wall compared with the platelets, which enables MPs separation into the inner outlet (MP outlet). The platelets and larger blood components are removed as waste. (b) Fluorescent and high speed images illustrating the equilibrium positions of the $2 \mu \mathrm{m}$ beads, blood cells and platelets. Inset (blue box) is a representative image of a platelet $(\sim 2-3 \mu \mathrm{m})$ at $\times 60$ magnification. (c) The physical characterization of MPs purified from endothelial cell culture (HUVECs) using dynamic light scattering (DLS) and fieldemission SEM (FESEM). The comparison of MPs separation efficiency between (d) HUVEC culture media and (e) whole blood samples. (f) Characterization of MPs recovery at different sample blood dilutions $\left({ }^{*} P<0.05\right.$ vs control $\left.(1: 5)\right)$. The data are presented as the mean \pm s.d. from $n=4$. 
sorted into the inner outlet (outlet 1) based on inertial focusing at the inner wall. A sample flow rate of $160 \mu \mathrm{min}^{-1}$ was optimized using $5 \mu \mathrm{m}$ particles to collect the larger PLGA particles $(>5 \mu \mathrm{m})$ (Supplementary Figure S4). Next, the outer outlet (outlet 2, containing smaller particles) was processed again by HiDFF at lower flow conditions $\left(\sim 80 \mu \mathrm{min}^{-1}\right)$ to retrieve the smallest particles from the inner outlet, whereas the medium-sized particles were collected into the outer outlet. The particle size and morphology were characterized using SEM, which confirmed the distinct size differences in each group (unsorted, $3.3 \pm 2.3 \mu \mathrm{m}$; large, $6.8 \pm 2.4 \mu \mathrm{m}$; medium, $1.7 \pm 1.0 \mu \mathrm{m}$; small, $0.89 \pm 0.46 \mu \mathrm{m}$ ) (Figure $3 \mathrm{c}$ ).

After HiDFF separation, we performed a drug release study by incubating equal weights of particles for each size group in a saline buffer. Distinct differences in the cumulative amounts of drug released were observed among the groups, which correlated inversely with the particle size (Figure 3d). The cumulative drug release was significantly higher for the smallest particles at day $10(20.97 \pm 1.82 \mu \mathrm{g}, P<0.01)$, followed by the medium size group $(4.01 \pm 0.57 \mu \mathrm{g}, P<0.05)$. The large and unsorted particles exhibited the least amount of cumulative release $(1.32 \pm 0.87 \mu \mathrm{g}$ and $1.75 \pm 0.17 \mu \mathrm{g}$, respectively). This was attributed to the increasing surface area to volume ratio for decreasing particle size, which enabled more efficient buffer penetration. ${ }^{28}$ Similarly, the drug release rate was the highest for the smallest particles, due to the shorter diffusion distance for the encapsulated drug (Figure 3c).

PTX is an Food and Drug Administration-approved drug for cancer treatment that induces mitotic arrest. ${ }^{30}$ To study the effect of particle size on inhibition of cancer cell proliferation, HiDFF-sorted PTXloaded PLGA MPs (100 nM) were incubated with a breast cancer cell line (MCF-7) for 4 days, and cell proliferation was assessed using a cell counting kit assay. PLGA MPs are highly biocompatible and the particle concentration used was in the reported range with low cytotoxicity. ${ }^{31,32}$ As expected, the largest particles were the least effective in inhibiting cell proliferation, as evidenced by the highest relative optical densities from days 2-4 (Figure 3e). Medium-sized particles had a similar cell proliferation profile as the unsorted sample, possibly due to their similar size ranges. The smallest group containing sub-micrometer particles exhibited the greatest inhibition of cell growth at days 3 and 4, which was significantly better than other particle size groups (relative optical density 0.74 (small) vs 0.95 (medium) and 1.07 (large) $P<0.01$ ), as well as the free drug at the same concentration. Taken together, these results clearly showed that the smallest drug-loaded MPs had enhanced drug release capabilities and HiDFF served as a useful tool for high-throughput fractionation of these sub-micrometer particles from a heterogeneous mixture for more effective drug treatment.

\section{Single-step isolation of circulating MPs from whole blood}

Circulating MPs in blood are of different cellular origins (platelets, blood cells and endothelium) and can affect a broad spectrum of biological activities including coagulation, inflammation and vascular homeostasis. Despite the widespread biological and clinical implications, the study of MPs is largely limited by their isolation from whole blood, which involves laborious, multi-step, high-speed centrifugation to deplete blood cells and platelets. ${ }^{11,13}$ Herein, we demonstrated direct single-step isolation of MPs from whole blood using HiDFF and characterized the device performance using human umbilical vascular endothelial cells and blood samples obtained from healthy donors.

Diluted whole blood $(1: 5 \mathrm{v} / \mathrm{v})$ and sheath fluid were introduced into the HiDFF device at the channel outer and inner wall, respectively. Under the influence of Dean vortices, MPs recirculated closer to the inner wall than the platelets did $(\sim 2-3 \mu \mathrm{m})$, which enabled MPs separation into the inner outlet (MP outlet), whereas platelets and larger blood components were removed as waste (Figure 4a). We first optimized the flow conditions based on $2 \mu \mathrm{m}$ fluorescent beads (comparable size to platelets), to ensure their complete separation into the waste outlet. Flow cytometry analysis of the eluent from the MP outlet indicated little platelet contamination during blood processing (Supplementary Figure S5). High-speed imaging clearly showed that the larger blood cells, experiencing significant Stoke's drag, remained closer to the outer wall at the outlet bifurcation (Figure 4b). The HiDFF-sorted MPs were then characterized using dynamic light scattering and nanoparticle tracking analysis, to confirm their size range $(0.1-1 \mu \mathrm{m})$, showing negligible differences in the MPs sizes when purified by centrifugation and HiDFF (Figure $4 \mathrm{c}$ and Supplementary Figure S6). Interestingly, field-emission SEM imaging revealed morphological differences between both methods; the HiDFF-purified MPs had more intact surface membranes as opposed to centrifuge-purified MPs, which exhibited more 'breakages' (Figure 4c). We hypothesize that the HiDFF is a gentler MPs separation approach, as sorted MPs are continuously recovered in the media with a single step, whereas centrifugation typically requires a longer duration and multiple steps of MPs pelleting $(20000 \mathrm{~g})$ and vortexing to form the suspension.

Using the developed technology, $46.6 \pm 2.77 \%$ MPs separation efficiency was achieved at the MP outlet for the human umbilical vascular endothelial cells culture media (Figure 4d), which was similar to whole blood samples $(52.8 \pm 4.53 \%$ ) (Figure $4 \mathrm{e}$ ), indicating that the presence of blood cell components did not affect the lateral migration of the MPs in the spiral device. This result was further confirmed as we varied the blood dilution and demonstrated that the HiDFF can process up to $\sim 20-25 \%$ hematocrit blood samples $(1: 1 \mathrm{v} / \mathrm{v})$ with complete RBCs removal, minimal platelet contamination and increased MPs recovery $(P<0.05$ vs control $(1: 5$ blood dilution $))$ at the MP outlet (Figure $4 \mathrm{f}$ and Supplementary Figure S5). This is important as it enables us to readily increase the blood processing throughput or shorten the processing time for downstream applications.

\section{Characterization of HiDFF-purified circulating MPs using flow cytometry}

To establish the use of HiDFF for MPs phenotyping, we isolated circulating MPs from whole blood of healthy subjects and performed downstream flow cytometry analysis to quantify EMPs ((CD144+ (vascular endothelial-cadherin), CD41a-)), LMPs (CD45+) and NMPs $(\mathrm{CD} 66 \mathrm{~b}+)$. Similar to MPs purified from culture media, HiDFF-purified MPs had well-preserved surface morphologies based on field-emission SEM imaging (Figure 5a). Crucially, negligible differences were observed in the quantities of various MPs subtypes (EMPs, LMPs and NMPs) between the HiDFF and centrifugation, which clearly indicated its potential as a single step MPs purification tool (Figure $5 \mathrm{~b}$ ). This trend was also consistent when comparing the isolation of lymphocyte-derived MPs (CD19+ and CD3+) using both methods (Supplementary Figure S7). Then, we evaluated the accuracy of the HiDFF-based MPs phenotyping by comparing the MPs count of the EMPs and the immune cell-derived MPs (CD45+, CD66b+, CD19 + and $\mathrm{CD} 3+)$ with centrifugation. A strong linearity $(\sim 1.25)$ and correlation $\left(R^{2}: 0.9\right)$ were observed between both MPs purification methods, demonstrating the feasibility of using HiDFF for rapid MPs quantification (Figure 5c).

Finally, we applied the developed technology to characterize circulating MPs in a cohort of T2DM subjects $((n=9)$, Supplementary Table S1). T2DM was used as the disease model 
a

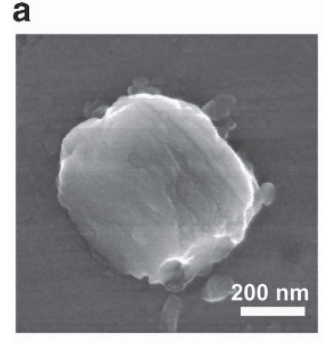

b

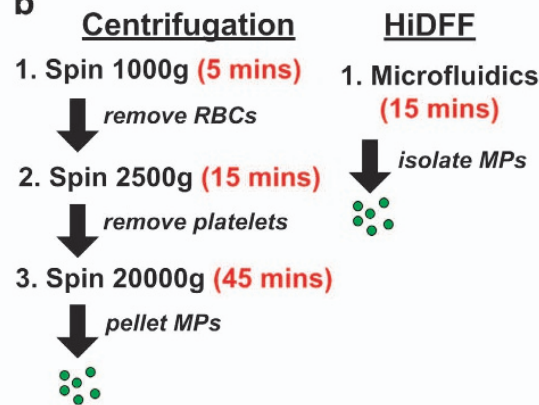

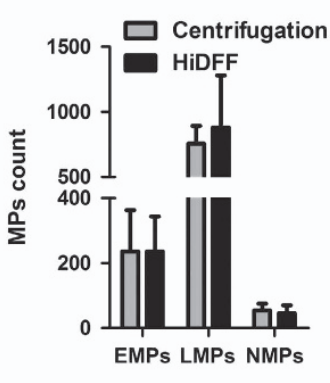

C

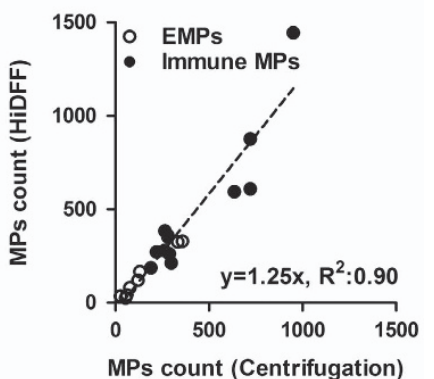

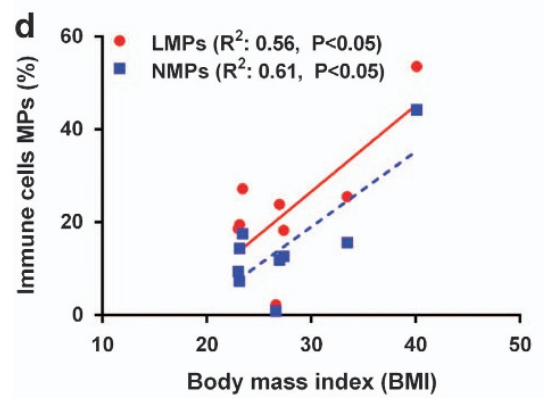
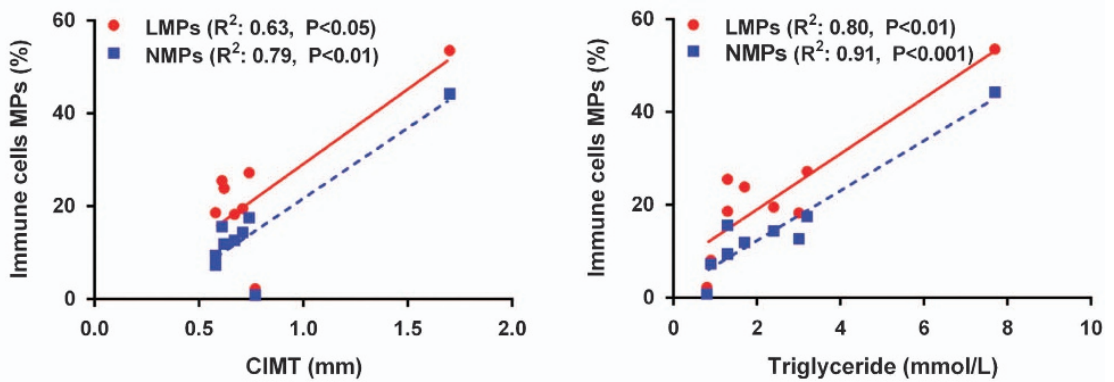

Figure 5 Characterization of the HiDFF-purified MPs using flow cytometry. (a) Representative field-emission SEM (FESEM) image of a HiDFF-purified MPs from whole blood. (b) Comparison of MPs isolation between the HiDFF- and centrifugation-based methods on EMPs (CD144+, CD41a-), LMPs (CD45+) and NMPs $(\mathrm{CD} 66 \mathrm{~b}+)$ in healthy subjects $(n=4)$. The data are presented as the mean \pm s.d. (c) There is a strong correlation in the MPs quantification (slope: $1.25, R^{2}$ : 0.9) between the centrifugation and HiDFF using EMPs and immune cell-derived MPs (CD45+, CD66b+, CD3+ and CD19+). (d) The association of HiDFF-purified LMPs and NMPs with body mass index (BMI), carotid intima-media thickness (CIMT) and triglyceride levels in T2DM patients ( $n=9$ ).

because of the high risk for vascular complications, and previous works have reported the pathological implications ${ }^{33,34}$ and clinical utilities $^{35,36}$ of circulating MPs in T2DM patients. Interestingly, both immune MPs (LMPs and NMPs) were strongly correlated to established cardiovascular risk factors, including body mass index, carotid intima-media thickness and triglyceride levels $(P<0.05$, $P<0.01$ and $P<0.001$, respectively) in T2DM patients (Figure 5d), suggesting a link between inflammation and lipid metabolism in diabetes patients. This link was further evident when we observed a negative association of the EMPs with the high-density lipoprotein cholesterol $(P<0.01)$ (Supplementary Figure S8). Overall, these results demonstrated the efficacy of HiDFF technology for rapid MPs sorting and phenotyping, and further studies are warranted to study the role of LMPs and NMPs in relation to lipid patterns and endothelial dysfunction in T2DM, as well as micro- and macrovascular disorders in general.

\section{DISCUSSION}

In this work, we report for the first time a unique Dean migration phenomenon in spiral inertial microfluidics where the particle innermost transient position $\left(D_{\text {inner }}\right)$ at the channel inner wall varies with particle size during lateral migration. This leads to the development of a novel separation technique (HiDFF) for continuous fractionation of engineered or biological small particles with high separation resolution $(\sim 1 \mu \mathrm{m})$ and throughput $\left(\sim 100 \mu \mathrm{min}^{-1}\right)$. We demonstrate the translational capabilities of HiDFF by rapid purification of submicrometer drug loaded particles for enhanced cancer treatment and single-step isolation of circulating MPs from whole blood for flow cytometry-based vascular health profiling. Compared with conventional separation approaches (membrane filtration and gradient centrifugation), the membrane-free microfluidics device is simple to operate and enables continuous collection of sorted biological targets from complex biosamples (for example, blood). This approach eliminates membrane clogging or fouling issues and can be easily multiplexed for high volume processing.

Inertial microfluidics is widely used as a passive, size-based sorting technique for cell separation $(\sim 8-20 \mu \mathrm{m}) .{ }^{37}$ However, inertial focusing of smaller particles $(\sim 1-2 \mu \mathrm{m})$ is more challenging due to the requirement for smaller channel geometries $\left(a_{\mathrm{p}} / D_{\mathrm{h}}>0.07\right.$; $D_{\mathrm{h}} \leq \sim 10 \mu \mathrm{m}$ for $1 \mu \mathrm{m}$ bead focusing) and the large channel resistance would cause flow-induced channel deformation. ${ }^{38}$ Bhagat et al. ${ }^{39}$ demonstrated shear-modulated inertial separation of $1.9 \mu \mathrm{m}$ and $590 \mathrm{~nm}$ particles using a straight rectangular microchannel, which has limited separation sensitivity. A spiral sorter was also developed to inertially focus $2.1 \mu \mathrm{m}$ and $3.2 \mu \mathrm{m}$ particles, but the operating flow rate was low $\left(10 \mu \mathrm{min}^{-1}\right)$ due to large pressure drop. ${ }^{40} \mathrm{HiDFF}$ addresses many of these limitations because it achieves particle separation solely based on the differential Dean migration profiles without inertial focusing. The throughput is significantly higher $\left(\sim 100 \mu \mathrm{min}^{-1}\right)$ with larger channel dimensions, which is more robust and suitable for processing complex biosamples (for example, blood) with minimal clogging.

In particle-based drug delivery systems, the polydispersity and batch-to-batch variation in particles are inherent to most of the particle fabrication techniques and have been linked to undesirable fluctuations in the degradation rate of particles, release rate of drugs and particle stability. ${ }^{7,41}$ In addition, although the smaller particles tend to cause undesirable burst releases, ${ }^{42}$ larger particles can pose problems in microcirculation due to their inability to travel through small blood capillaries. The capability to segregate polydisperse particles into different size ranges is highly useful for in vivo drug delivery studies and organ-specific applications. For example, drug delivery across the highly regulated blood-brain barrier requires lipidsoluble particles $<200 \mathrm{~nm}$. ${ }^{43}$ In contrast, the effective particle size for 
pulmonary drug administration is $\sim 1-5 \mu \mathrm{m}$, as smaller particles $(<1 \mu \mathrm{m})$ are absorbed from the airways before reaching the lungs, therefore posing a risk for systemic toxicity. ${ }^{44}$ By controlling the flow rate and channel outlet resistance, HiDFF offers simple and tunable size fractionation of polydisperse drug-loaded particles into different size groups using the same device. This not only enables efficient biodistribution and controlled drug release with distinct particle sizes, but will potentially reduce cost and toxicity with lower usage of more potent, smaller particles.

Despite the significant interest in circulating extracellular vesicles (MPs and exosomes) in blood, clinical utility is limited due to their small sizes $(<1 \mu \mathrm{m})$ and isolation difficulties. ${ }^{11}$ High-speed centrifugation or ultracentrifugation is generally considered the gold standard for MPs isolation, but it remains a challenge for low sample volumes $(\sim 100-500 \mu \mathrm{l})$. Moreover, there is a risk of active MPs release from blood cells and platelets during processing. ${ }^{45} \mathrm{~A}$ unique advantage of HiDFF is the size-based purification of MPs with the complete removal of platelets $(\sim 2-3 \mu \mathrm{m})$ and blood cells $(\sim 8-15 \mu \mathrm{m})$ in a single step. This label-free approach enables unbiased analysis of circulating MPs and is significantly less expensive compared with antibody-based techniques. As HiDFF is designed to isolate submicrometer microvesicles, it will isolate both exosomes $(40-100 \mathrm{~nm})$ and MPs (100 nm to $1 \mu \mathrm{m}$ ) simultaneously from blood and additional ultra-centrifugation steps are necessary to isolate pure exosomes. Compared with centrifugation, the developed method is also gentler as it can preserve the MPs morphology (less surface 'breakage') with its rapid isolation process and minimal user manipulation or post processing. Current work aims to determine whether these morphological differences have a role in proteomics studies or cell-cell communications.

Clinically, endothelial functions are assessed by invasive and noninvasive methods based on indirect measurement of the vascular response to vasodilation or reactive hyperemia ${ }^{46}$ and the accuracy of these methods will be greatly improved with new quantitative biomarkers and techniques. Circulating MPs are an attractive alternative, because they are implicated in vascular homeostasis ${ }^{47,48}$ and can be readily characterized with liquid biopsy. We first quantified circulating MPs using flow cytometry and showed a good correlation between HiDFF and centrifugation MPs isolation methods. As proofof-concept for clinical testing, we applied our HiDFF-based approach to analyze MPs phenotype in T2DM patients. Both LMPs and NMPs levels are strongly correlated with the body mass index, carotid intimamedia thickness and triglyceride levels, which are established cardiovascular risk factors and related to vascular inflammation. ${ }^{49}$ As immune MPs can mediate inflammatory response and endothelial functions,${ }^{50,51}$ our data suggest the potential of using circulating MPs as surrogate biomarkers for endothelial function testing.

In summary, there exists a critical and unmet need to develop novel tools for efficient and high throughput separation of sub-micrometer particles, as exemplified by two major clinical problems in cancer therapy and vascular profiling. HiDFF represents an important technological progress towards small engineered or biological target purification with its membrane-free, passive, size-based separation approach that facilitates scaling up for high volume processing. Finally, as HiDFF enables direct whole blood processing and rapid label-free isolation of MPs, it can be readily integrated with other detection assays, and further developed into a clinical tool for quantitative measurement of vascular dysfunction.

\section{CONFLICT OF INTEREST}

The authors declare no conflict of interest.

\section{ACKNOWLEDGEMENTS}

BOB is supported by the Lee Kong Chian School of Medicine, Nanyang Technological University Start Up Grant, MOE AcRF Tier 1 (2015-T1-001-258) and Nanyang Technological University-National Healthcare Group (NTUNHG) Metabolic Diseases Collaboration Grant (MDCG/15006). SCJL acknowledges the financial support from the Singapore Centre on Environmental Life Sciences Engineering (SCELSE) (M4330001.C70.703012), the School of Materials Science and Engineering (M020070110), the Ministry of Health (NMRC/CIRG/1342/2012, MOH) and the NTU-NHG grant (ARG/ 14012). HWH acknowledges the financial support from the Lee Kong Chian School of Medicine Postdoctoral Fellowship, the NTU-NHG Metabolic Diseases Collaboration Grant (MDCG/15004) and the Singapore National Research Foundation under CBRG NIG, and administered by the Singapore Ministry of Health's National Medical Research Council (NMRC-08/2015BNIG).

Author contributions: HWH, RD and SCJL conceived the idea and designed the research. HMT, HWH, SK, ZJC and KKT performed the experiments and analyzed the data. $\mathrm{RD}$ and $\mathrm{BOB}$ assisted in patient recruitment, clinical analysis and evaluation of the translational capabilities of the developed technology. HMT, SK, SCJL and HWH wrote the manuscipt. All authors reviewed the manuscript.

\section{PUBLISHER'S NOTE}

Springer Nature remains neutral with regard to jurisdictional claims in published maps and institutional affiliations.

1 Farokhzad, O. C. \& Langer, R. Impact of nanotechnology on drug delivery. ACS Nano 3 , 16-20 (2009).

2 Raposo, G. \& Stoorvogel, W. Extracellular vesicles: exosomes, microvesicles, and friends. J. Cell Biol. 200, 373-383 (2013).

3 Müller, K., Fedosov, D. A. \& Gompper, G. Margination of micro- and nano-particles in blood flow and its effect on drug delivery. Sci. Rep. 4, 4871 (2014).

4 Siepmann, J., Faisant, N., Akiki, J., Richard, J \& Benoit, J. P. Effect of the size of biodegradable microparticles on drug release: experiment and theory. J. Control. Release 96, 123-134 (2004).

5 Rolland, J. P., Maynor, B. W., Euliss, L. E., Exner, A. E., Denison, G. M. \& DeSimone, J. $M$. Direct fabrication and harvesting of monodisperse, shape-specific nanobiomaterials. J. Am. Chem. Soc. 127, 10096-10100 (2005).

6 Guan, J., Ferrell, N., James Lee, L. \& Hansford, D. J. Fabrication of polymeric microparticles for drug delivery by soft lithography. Biomaterials 27 , 4034-4041 (2006).

7 Xu, Q., Hashimoto, M., Dang, T. T., Hoare, T, Kohane, D. S., Whitesides, G. M., Langer R. \& Anderson, D. G. Preparation of monodisperse biodegradable polymer microparticles using a microfluidic flow-focusing device for controlled drug delivery. Small 5, 1575-1581 (2009).

8 Rhee, M., Valencia, P. M., Rodriguez, M. I., Langer, R., Farokhzad, O. C. \& Karnik, R. Synthesis of size-tunable polymeric nanoparticles enabled by 3D hydrodynamic flow focusing in single-layer microchannels. Adv. Mater. 23, H79-H83 (2011).

9 Iero, M., Valenti, R., Huber, V., Filipazzi, P., Parmiani, G., Fais, S. \& Rivoltini, L. Tumour-released exosomes and their implications in cancer immunity. Cell Death Differ. 15, 80-88 (2007).

10 Müller, G. Microvesicles/exosomes as potential novel biomarkers of metabolic diseases. Diabetes Metab. Syndr. Obes. Target Ther. 5, 247-282 (2012).

11 Théry, C., Amigorena, S., Raposo, G. \& Clayton, A. Isolation and characterization of exosomes from cell culture supernatants and biological fluids. Curr. Protoc. Cell Biol. Chapter 3, Unit 3.22, 1-29 (2001).

12 Tauro, B. J., Greening, D. W., Mathias, R. A., Ji, H., Mathivanan, S., Scott, A. M. \& Simpson, R. J. Comparison of ultracentrifugation, density gradient separation, and immunoaffinity capture methods for isolating human colon cancer cell line LIM1863derived exosomes. Methods 56, 293-304 (2012).

13 Shet, A. S. Characterizing blood microparticles: technical aspects and challenges. Vasc. Health Risk Manag. 4, 769-774 (2008).

14 Chen, C., Skog, J., Hsu, C. H., Lessard, R. T., Balaj, L., Wurdinger, T., Carter, B. S., Breakefield, X. O., Toner, M \& Irimia, D. Microfluidic isolation and transcriptome analysis of serum microvesicles. Lab Chip 10, 505-511 (2010).

15 Davies, R. T., Kim, J., Jang, S. C., Choi, E. J., Gho, Y. S. \& Park, J. Microfluidic filtration system to isolate extracellular vesicles from blood. Lab Chip 12, 5202-5210 (2012).

16 Zhao, Z., Yang, Y., Zeng, Y. \& He, M. A microfluidic ExoSearch chip for multiplexed exosome detection towards blood-based ovarian cancer diagnosis. Lab Chip 16, 489-496 (2016).

17 Bhagat, A. A. S., Bow, H., Hou, H. W., Tan, S. J., Han, J. \& Lim, C. T. Microfluidics for cell separation. Med. Biol. Eng. Comput. 48, 999-1014 (2010). 
18 Huang, L. R., Cox, E. C., Austin, R. H. \& Sturm, J. C. Continuous particle separation through deterministic lateral displacement. Science 304, 987-990 (2004).

19 Yamada, M. \& Seki, M. Hydrodynamic filtration for on-chip particle concentration and classification utilizing microfluidics. Lab Chip 5, 1233-1239 (2005).

20 Petersson, F., Åberg, L., Swärd-Nilsson, A.-M. \& Laurell, T. Free flow acoustophoresis: microfluidic-based mode of particle and cell separation. Anal. Chem. 79, 5117-5123 (2007)

21 Di Carlo, D., Irimia, D., Tompkins, R. G. \& Toner, M. Continuous inertial focusing, ordering, and separation of particles in microchannels. Proc. Natl Acad. Sci. USA 104 18892-18897 (2007)

22 Hou, H. W., Warkiani, M. E., Khoo, B. L., Li, Z. R., Soo, R. A., Tan, D. S. W., Lim, W. T., Han, J., Bhagat, A. A. S. \& Lim, C. T. Isolation and retrieval of circulating tumor cells using centrifugal forces. Sci. Rep. 3, 1259 (2013).

23 Hou, H. W., Bhattacharyya, R. P., Hung, D. T. \& Han, J. Direct detection and drug resistance profiling of bacteremias using inertial microfluidics. Lab Chip 15 2297-2307 (2015).

24 Yeo, D. C., Wiraja, C., Zhou, Y., Tay, H. M., Xu, C. J. \& Hou, H. W. Interference-free micro/nanoparticle cell engineering by use of high-throughput microfluidic separation. ACS Appl. Mater. Interfaces 7, 20855-20864 (2015).

25 Birch, C. M., Hou, H. W., Han, J. \& Niles, J. C. Identification of malaria parasiteinfected red blood cell surface aptamers by inertial microfluidic SELEX (I-SELEX). Sci. Rep. 5, 11347 (2015).

26 Ookawara, S., Street, D. \& Ogawa, K. Numerical study on development of particle concentration profiles in a curved microchannel. Chem. Eng. Sci. 61, 3714-3724 (2006)

27 Stone, H. A. Philip Saffman and viscous flow theory. J. Fluid Mech. 409, 165-183 (2000)

28 Dunne, M., Corrigan, O. I. \& Ramtoola, Z. Influence of particle size and dissolution conditions on the degradation properties of polylactide-co-glycolide particles. Biomaterials 21, 1659-1668 (2000).

29 Yin Win, K. \& Feng, S.-S. Effects of particle size and surface coating on cellular uptake of polymeric nanoparticles for oral delivery of anticancer drugs. Biomaterials 26 , 2713-2722 (2005)

30 McGuire, W. P., Rowinsky, E. K., Rosenshein, N. B., Grumbine, F. C., Ettinger, D. S., Armstrong, D. K. \& Donehower, R. C. Taxol: a unique antineoplastic agent with significant activity in advanced ovarian epithelial neoplasms. Ann. Intern. Med. 111, 273-279 (1989).

31 Xiong, S., George, S., Yu, H., Damoiseaux, R., France, B., Ng, K. W. \& Loo, J. S. Size influences the cytotoxicity of poly (lactic-co-glycolic acid) (PLGA) and titanium dioxide (TiO2) nanoparticles. Arch. Toxicol. 87, 1075-1086 (2013).

32 de Lima, R., do Espirito Santo Pereira, A., Porto, R. M. \& Fraceto L, F Evaluation of cyto- and genotoxicity of poly(lactide-co-glycolide) nanoparticles. J. Polym. Environ. 19, 196-202 (2011)

33 Tramontano, A. F., Lyubarova, R., Tsiakos, J., Palaia, T., Deleon, J. R. \& Ragolia, L. Circulating endothelial microparticles in diabetes mellitus. Mediat. Inflamm. 2010 250479 (2010)

34 Sabatier, F., Darmon, P., Hugel, B., Combes, V., Sanmarco, M., Velut, J. G., Arnoux, D., Charpiot, P., Freyssinet, J. M., Oliver, C., Sampol, J \& Dignat-George, F. Type 1 and type 2 diabetic patients display different patterns of cellular microparticles. Diabetes 51, 2840-2845 (2002).

35 Curtis, A. M., Zhang, L., Medenilla, E., Gui, M., Wilkinson, P. F., Hu, E., Giri, J., Doraiswamy, V., Gunda, S., Burgert, M. E., Moore, J. S., Edelberg, J. M. \& Mohler, E. R. III Relationship of microparticles to progenitor cells as a measure of vascular health in a diabetic population. Cytometry B Clin. Cytom. 78B, 329-337 (2010).

36 Nozaki, T., Sugiyama, S., Koga, H., Sugamura, K., Ohba, K., Matsuzawa, Y., Sumida, H., Matsui, K., Jinnouchi, H. \& Ogawa, H. Significance of a multiple biomarkers strategy including endothelial dysfunction to improve risk stratification for cardiovascular events in patients at high risk for coronary heart disease. J. Am. Coll. Cardiol. 54 601-608 (2009).

37 Di Carlo, D. Inertial microfluidics. Lab Chip 9, 3038-3046 (2009)

38 Sollier, E., Murray, C., Maoddi, P. \& Di Carlo, D. Rapid prototyping polymers for microfluidic devices and high pressure injections. Lab Chip 11, 3752-3765 (2011)

39 Bhagat, A., Kuntaegowdanahalli, S. \& Papautsky, I. Inertial microfluidics for continuous particle filtration and extraction. Microfluidics Nanofluidics 7, 217-226 (2009).

40 Johnston, I. D., McDonnell, M. B., Tan, C. K. L., McCluskey, D. K., Davies, M. J. \& Tracey, M. C. Dean flow focusing and separation of small microspheres within a narrow size range. Microfluidics Nanofluidics 17, 509-518 (2014).

41 Sansdrap, P. \& Moës, A. J. Influence of manufacturing parameters on the size characteristics and the release profiles of nifedipine from poly(DL-lactide-co-glycolide) microspheres. Int. J. Pharm. 98, 157-164 (1993).

42 Berchane, N. S., Carson, K. H., Rice-Ficht, A. C. \& Andrews, M. J. Effect of mean diameter and polydispersity of PLG microspheres on drug release: experiment and theory. Int J. Pharm. 337, 118-126 (2007).

43 Lockman, P. R., Mumper, R. J., Khan, M. A. \& Allen, D. D. Nanoparticle technology for drug delivery across the blood-brain barrier. Drug Dev. Ind. Pharm. 28, 1-13 (2002)

44 Dhand, C., Prabhakaran, M. P., Beuerman, R. W., Lakshminarayanan, R., Dwivedid, N. \& Ramakrishna, S. Role of size of drug delivery carriers for pulmonary and intravenous administration with emphasis on cancer therapeutics and lung-targeted drug delivery. RSC Adv. 4, 32673-32689 (2014).

45 Simak, J. \& Gelderman, M. P. Cell membrane microparticles in blood and blood products: potentially pathogenic agents and diagnostic markers. Transfus. Med. Rev. 20, 1-26 (2006)

46 Kuvin, J. T., Patel, A. R., Sliney, K. A., Pandian, N. G., Sheffy, J., Schnall, R. P., Karas, R. H. \& Udelson, J. E. Assessment of peripheral vascular endothelial function with finger arterial pulse wave amplitude. Am. Heart J. 146, 168-174 (2003).

47 Dignat-George, F. \& Boulanger, C. M. The many faces of endothelial microparticles. Arterioscler. Thromb. Vasc. Biol. 31, 27-33 (2011).

48 Boulanger, C. M., Scoazec, A., Ebrahimian, T., Henry, P., Mathieu, E., Tedgui, A. \& Mallat, Z. Circulating microparticles from patients with myocardial infarction cause rndothelial dysfunction. Circulation 104, 2649-2652 (2001).

49 Gordillo-Moscoso, A., Carnero, M., Reguillo, F., Rodriguez, E., Tejerina, T. \& Redondo, S. Relationship between serum levels of triglycerides and vascular inflammation, measured as COX-2, in arteries from diabetic patients: a translational study. Lipids Health Dis. 12, 62 (2013).

50 Angelillo-Scherrer, A. Leukocyte-derived microparticles in vascular homeostasis. Circ. Res. 110, 356-369 (2012).

51 Mesri, M. \& Altieri, D. C. Endothelial cell activation by leukocyte microparticles. J. Immunol. 161, 4382-4387 (1998).

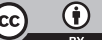

This work is licensed under a Creative Commons Attribution 4.0 International License. The images or other third party material in this article are included in the article's Creative Commons license, unless indicated otherwise in the credit line; if the material is not included under the Creative Commons license, users will need to obtain permission from the license holder to reproduce the material. To view a copy of this license, visit http:// creativecommons.org/licenses/by/4.0/

(C) The Author(s) 2017

Supplementary Information accompanies the paper on the NPG Asia Materials website (http://www.nature.com/am) 